\title{
Design and evaluation of a breath analysis system for occupational exposure assessment
}

\author{
K. L. Soldat and K. D. Thrall \\ Pacific Northwest National Laboratory, United States of America
}

\begin{abstract}
Exposure assessment is an integral part of industrial hygiene and occupational health. To ensure the health and safety of workers, integrated industrial hygiene methodologies often include biological monitoring strategies. Exhaled breath is an ideal matrix for measuring volatile biomarkers, particularly since the noninvasive collection of breath may improve volunteer participation. A real-time, field-portable system was developed to analyze undiluted exhaled air from experimental animals and humans. The system combines 1) an ion-trap mass spectrometer capable of atmospheric sampling; 2) a breath interface for continual analysis of the exhaled breath stream; 3) chemical dosimeters that are analyzed in the field/workplace; and 4) physiologically based pharmacokinetic (PBPK) models to estimate total exposure and internal target tissue dosimetry. The intent of this development was to provide new instrumentation to evaluate volatile chemical exposures as part of a daily monitoring program. For example, the system was designed to monitor a worker every time they enter and leave a work environment - a vast improvement over current 8-hr integrated monitoring strategies. To evaluate the system in actual work environments, field tests were conducted using volunteers providing exhaled breath samples before and after each specific job task. In these field studies, several volunteers had post-task breath levels higher than pre-task levels. Compared to the breath analysis findings, chemical dosimeters underpredicted exposures, particularly for longer sampling intervals where the volume of air sampled may have diluted exposures. The results of these field studies illustrate the utility of monitoring workers for exposures at numerous times throughout the day, particularly when job-specific tasks may indicate a potential for exposure.
\end{abstract}




\section{Introduction}

A comprehensive worker surveillance program designed to reduce or prevent workplace exposures generally includes job task analysis, exposure assessment, hazard surveillance, medical surveillance, and epidemiologic surveillance. Often, exposure assessment is the weakest link in this program. With few exceptions, traditional methods of exposure assessment have consisted of ambient air monitoring using stationary or personal samplers to measure the air concentration of a chemical. However, monitoring the ambient air fails to assess the exposure of the individual worker, and personal samplers fail to account for routes of exposure other than by inhalation, for example, by dermal or accidental ingestion pathways. Furthermore, a significant shortfall in ambient monitoring is that information is provided only on exposure, and not on the amount of a chemical that has effectively entered the body.

In contrast to exposure assessment, a biomarker can provide valuable information in the assessment of exposure to hazardous materials in the individual workers. Unlike air monitoring, biological monitoring of exposure integrates exposures from all likely routes and can account for nonoccupational exposures as well, such as from smoking, using household products, and recreational activities that may result in exposures. In industrial hygiene, the assessment of a biomarker of exposure, referred to as a biological exposure indice (BEI), provides a useful measurement of internalized exposure. The BEI represents the amount of chemical that would be in a biological sample, such as blood, urine, or exhaled breath, collected from a worker exposed by inhalation at the threshold limit value (TLV) concentration, eight hours a day, five days a week.

To be useful, a BEI has to be detectable in a biological matrix at levels that correlate with permissible occupational exposure levels (sensitivity). A BEI must also be specific for an exposure, and not measurable after exposure to other chemicals (specificity). Together, sensitivity and specificity eliminate false negatives, chemical overlap, and false positives. In addition, the analytical technique used to measure the BEI must have low levels of variability in order to distinguish analytical noise from variability inherent to monitoring a nonhomogeneous population of workers. Finally, a BEI should be measurable in a biological sample that is easily collected, such as blood, urine, saliva, or exhaled breath.

The exhaled breath matrix for biological monitoring of occupational exposure offers a number of advantages over other matrices, including blood and urine. Exhaled breath is simple and fast to collect, and is collected in a completely noninvasive manner. In addition, expired air is a less complex matrix for analysis compared to either blood or urine, and avoids contact with potentially infectious waste. Despite these advantages, exhaled breath analysis has been of limited use in routine monitoring of occupational exposures (Ljungkvist and Nordlinder, 1995). The major criticism regarding the use of exhaled breath as a BEI is that the timing of sample collection is critical to evaluate the degree of exposure. Part of this may be due to the lack of field deployable methods that are simple and reliable, and due to the absence of standard monitoring protocols. Standardization is especially 
important for volatile compounds, as the rapid clearance from the lung makes breath measurements highly dependent of the time of sampling following exposure.

In support of the mission of the Department of Energy (DOE), a field-portable breath-analysis system has been developed to analyze undiluted exhaled air from humans in real-time (Thrall and Kenny, 1998; Thrall et al. 2001). Because monitoring is non-invasive and the analysis is conducted in real-time, the system can be used to monitor chemical exposures of individuals every time they enter and leave a hazardous area. A physiologically based pharmacokinetic (PBPK) model is used to interpret the exhaled breath data and calculate an internal, target tissue dose based on the kinetics of elimination in the exhaled breath. The design of the system, and field studies evaluating the utility of the system in an occupational environment are described here.

\section{Monitoring system development}

\subsection{Breath analysis}

The exhaled breath stream is monitored using a tandem ion-trap mass spectrometer (MS/MS) equipped with an atmospheric sampling glow discharge ionization (ASGDI) source. The MS/MS allows for detection limits in the 1-10 parts-perbillion ( $\mathrm{ppb}$ ) range for most volatile solvents encountered occupationally, and the ASGDI source allows for real-time analysis without regulating pressures between the exhaled breath and the ion-trap. In use, a worker is connected directly with the MS/MS system by exhaling through a disposable one-way breathing valve attached to a heated large-diameter transfer line into a heated glass mixing chamber (1.3 liters). The breath sample enters the glass mixing chamber via a tube that bends off to one side, and is pushed out of the mixing chamber by subsequent breaths via a tube that bends in the opposite direction. The bent tubes ensure that air samples are well mixed by turbulent flow, and the MS/MS system continually draws a sample from the center of the mixing chamber at a calibrated rate of $200 \mathrm{ml} / \mathrm{min}$. Intensity data from the MS/MS is converted to concentration (ppb) using external gas standards prepared in Tedlar bags and a calibration curve for each compound. A new calibration curve is generated each day of operation.

\subsection{Physiologically Based Pharmacokinetic (PBPK) Models}

A compound detected in the exhaled breath can be related to total exposure through the use of PBPK modeling. These physiologically relevant models are powerful tools that can be used to simulate a chemical exposure regardless of route, and estimate internal target tissue dose. A PBPK model is based on physiological parameters, chemical-specific parameters, and biochemical factors. The physiological information (tissue or organ volumes, blood flow rates, breathing rates, etc.) is well understood, and can be easily modified on a worker-by-worker basis. Chemical-specific parameters (partition coefficients, density, protein binding characteristics, etc.), and biochemical factors (metabolic rate constants, etc.) are 
either available in the literature, or can be developed by focused in vitro or in vivo laboratory studies. A generic PBPK model is illustrated in Figure 1.

The idea behind a PBPK model is to construct a series of differential equations that mathematically describe the absorption, distribution, metabolism, and elimination of a compound in defined tissue compartments. For each compartment, a compound carried in the arterial blood enters the tissue at a physiologically known flow rate, a portion of that compound is retained in the compartment based on its partition coefficient and the capacity for that tissue to metabolize the compound, and the remainder leaves the compartment via the venous blood and is recirculated. As the blood passes through the lung, a fraction of the compound is exhaled, depending on the blood to air partition coefficient. Given enough data, a PBPK model has the ability to predict parent or metabolite levels in blood or tissues and the amount eliminated in urine, feces, or exhaled breath following exposures by any route. The greatest strength in a well-developed PBPK model is that it can account for known differences in personal and physiological parameters and can be used on an individual-by-individual basis. Thus, by monitoring the exhaled breath for a particular compound, the estimated exposure and target tissue dose can be determined. This provides a powerful enhancement to occupational exposure assessment surveillance programs.

\subsection{Personal samplers}

In conjunction with monitoring exhaled breath, environmental exposures are assessed using more traditional industrial hygiene methodologies. For ambient air monitoring, an air sampling pump drew air through a chemical lapel absorbent stainless steel prepacked Tenax cartridge (SupelCo) at a calibrated flow rate. During field studies, volunteers wore a sampling pump each data and exchanged the absorbent tube for a clean tube each time they provided a breath sample. The time of sampling and flow rates were recorded to calculate total volume of air sampled. Tenax sample tubes were analyzed using a front-end thermal desorption device coupled to the field-portable MS/MS. Blank tubes and blank tubes spiked with known concentrations of chemicals are used to calibrate the system. In initial field studies volunteers wore sampling pumps with Tenax sampling tubes and charcoal sampling tubes (SKC-brand coconut shell charcoal 20/40 mesh) to allow for direct comparison on analytical methodology.

\section{Application in field studies}

A field study was conducted using the monitoring system to quantitate exposures to benzene and toluene at a waste incinerator plant. Exhaled breath samples were collected from volunteers prior to starting a particular job task, and again at the end of that job. Tasks ranged from 15 minutes to unload a waste drum, to 4-hours in the control room, and the frequency at which volunteers provided breath samples was dependent on the job task and the potential for exposure. Both toluene and benzene were simultaneously analyzed for concentration in the exhaled breath samples. Pre-activity breath samples were considered to reflect background 
concentrations, therefore job task-specific exposures were calculated as the difference between "post"- and "pre"-activity measurements.

\subsection{Volunteers}

Field studies were conducted under approval from the Pacific Northwest National Laboratory human subjects Institutional Review Board, in compliance with multiple project assurance number DOE.MPA.PNNL96-2000. Prior to participation all potential volunteers were briefed on the monitoring system and detection capabilities and given the opportunity to participate.

\section{Results}

In total there were 17 pairs of Tenax and charcoal sampling tubes collected for direct comparison of the analysis of the Tenax sampling tube using the front end on the ASGDI-MS/MS and gas chromatographic analysis of the charcoal tube. The gas chromatography method for the charcoal tubes had a limit of detection of 0.047 $\mu \mathrm{g} / \mathrm{ml}$ for toluene and $0.034 \mu \mathrm{g} / \mathrm{ml}$ for benzene. None of the charcoal tubes were found to have toluene or benzene concentrations above the detection limit of the analytical method. In contrast, both benzene and toluene were measured on all Tenax tubes (Table 1). In addition, evaluation of the charcoal tubes by a certified method required a more time intensive solvent extraction followed by gas chromatographic analysis, which delayed access to the exposure information. Pump failure occurred on 3 occasions, most commonly due to low batteries.

Exhaled breath samples were analyzed for a total of 22 comparative (pre- versus post-job task) times (Table 1). Every study participant had some level of toluene and benzene in their exhaled breath in the "pre"-activity phase. This is consistent with previous studies showing background levels of benzene in the exhaled breath of non-occupationally exposed individuals ranging from 8 to $20 \mathrm{ppb}$ (Wallace 1989). In over half the comparative cases, post-activity concentrations of toluene and/or benzene were lower than pre-activity levels (Figure 2).

The highest concentration of benzene measured in the exhaled breath was from a smoker participating in the field study, and corresponded to an increase over pretask levels of $23.9 \mathrm{ppb}$ (Sample No. 2-2). The highest concentration of toluene was an increase of $59.6 \mathrm{ppb}$ over pre-task levels and corresponded to a volunteer accidentally sprayed with fuel oil during a routine job. This worker provided a breath sample for analysis less than 5 minutes after the accidental spraying occurred. The toluene PBPK model predicts that a worker exposed to toluene by inhalation at the TLV of $50 \mathrm{ppm}$ for an 8-hour workday would have approximately $5 \mathrm{ppm}$ in the exhaled breath immediately at the end of exposure. In comparison, the exhaled breath concentration in the volunteer accidentally sprayed with fuel oil was roughly 80 times lower than predicted at the TLV level. 
Transactions on Biomedicine and Health vol 5, (c) 2001 WIT Press, www.witpress.com, ISSN 1743-3525

Table 1: Analysis of Tenax Samples Tubes and Exhaled Breath for Toluene and Benzene Exposures

\begin{tabular}{|l|c|c|c|c|}
\hline \multirow{2}{*}{$\begin{array}{l}\text { Sample } \\
\text { No. }\end{array}$} & \multicolumn{2}{|c|}{ Benzene (ppb) } & \multicolumn{2}{c|}{ Toluene (ppb) } \\
\cline { 2 - 5 } & Tenax & Breath & Tenax & Breath \\
\hline $1-2$ & N/A & $(6.2)^{\mathrm{b}}$ & N/A & $(6.7)$ \\
\hline $1-3$ & N/A & $(11.5)$ & N/A & $(6.7)$ \\
\hline $2-3 \mathrm{a}$ & 0.7 & 0.4 & 2.21 & 4.6 \\
\hline $2-3 \mathrm{~b}$ & 0.1 & 1.0 & 0.31 & $(3.4)$ \\
\hline $2-3 \mathrm{c}$ & 0.6 & 0.6 & 2.25 & 0 \\
\hline $2-2$ & 1.4 & 23.9 & 2.10 & $(9.4)$ \\
\hline $2-4$ & N/A & 2.6 & N/A & 6.6 \\
\hline $3-4$ & 0.7 & N/A & 1.16 & N/A \\
\hline $3-3$ & 0.4 & $(0.2)$ & 0.45 & $(0.7)$ \\
\hline $3-2$ & 0.9 & 6.4 & 0.39 & $(5.7)$ \\
\hline $4-6$ & 0.3 & $(0.4)$ & 0.42 & 3.9 \\
\hline $4-3$ & 0.4 & $(2.8)$ & 0.73 & $(0.8)$ \\
\hline $5-1$ & 0.9 & $(1.0)$ & 1.81 & 2.6 \\
\hline $5-3$ & 0.1 & 2.0 & 1.37 & N/A \\
\hline $5-5 \mathrm{a}$ & 4.2 & 1.1 & 9.44 & 5.5 \\
\hline $5-5 \mathrm{~b}$ & 17.3 & 2.6 & 8.87 & 4.8 \\
\hline $6-3$ & 5.3 & $(0.5)$ & 7.57 & 6.4 \\
\hline $6-5$ & 3.8 & $(1.6)$ & 1.61 & 5.0 \\
\hline $7-3$ & 19.2 & 12.0 & 8.82 & 59.6 \\
\hline $7-5$ & 17.8 & 3.9 & 5.61 & 18.1 \\
\hline $7-1$ & 11.3 & 1.6 & 5.60 & 10.9 \\
\hline $8-3$ & 1.4 & $(1.8)$ & 3.96 & 3.5 \\
\hline $8-5$ & 3.5 & $(2.4)$ & 6.51 & 0.6 \\
\hline
\end{tabular}

${ }^{\mathrm{a}} \mathrm{N} / \mathrm{A}$ - Not available because of sampling pump failure.

${ }^{b}$ Values (ppb) represent the difference between pre- and post-exposure monitoring. Values in parentheses are negative values, where the pre-exposure measurement is greater than the post-exposure measurement.

The PBPK models can be used to predict exhaled breath concentrations in volunteers at the exposure levels indicated from analysis of the Tenax cartridges. In some cases, the predicted breath levels compare quite well with measured breath concentrations (i.e., Figure 3). However, in most cases there was no correlation between the Tenax-measured exposure concentration and the amount of compound in the exhaled breath. Generally, the Tenax tube analyses tended to underpredict the exposure amount required to match the increased breath concentrations between pre- and post-activity measurements. For example, analysis of the Tenax tube for the study participant accidentally sprayed with fuel oil was $8.82 \mathrm{ppb}$ collected during 82 minutes of sampling. The PBPK model estimate of the amount of 
inhalation exposure necessary to match the increase of $59.6 \mathrm{ppb}$ in exhaled breath for this individual is $3 \mathrm{ppm}$ in the air. Since the accidental spraying incident occurred less than 5 minutes before a breath sample was provided, the volume of air sampled by the Tenax cartridge throughout the 82 minute sampling period may have diluted out a peak exposure such as indicated here.

\section{Discussion}

Exposure assessment is a critical component of industrial hygiene and worker health protection programs. Given today's diverse work environments, new and innovative methodologies for exposure assessment are needed in order to fully understand the potential health risks of the individual worker. Routine analysis of exhaled breath may be ideal for tracking occupational exposures, particularly with the advent of field-deployable standardized methodologies. Exhaled breath analysis offers a number of advantages, including being noninvasive, is applicable to a number of compounds, avoids the handling of potentially infectious biological samples, and can be analyzed easily and quickly using the methodology described here. Furthermore, the system described here goes well beyond traditional industrial hygiene exposure assessment methodologies by employing PBPK models to understand the relationship between exposure and internal, target tissue dose.

The field-portable monitoring system described here has been successfully transported to various locations around the United States on over a dozen separate occasions. These field studies have, for the most part, been conducted to assess the prototype system design in a working environment and to evaluate worker acceptability of system. While additional efforts at miniaturization, automation, and software integration continue to evolve based on these field studies, workers, field supervisors, and management have tended to respond that analysis left them feeling assured that no significant exposures had occurred. The field study described here illustrates the utility of monitoring workers for exposures at numerous times throughout the day, particularly when job-specific tasks may indicate a greater potential for exposure.

\section{Acknowledgments}

Various aspects of this research were supported by the U.S. Department of Energy under Contract DE-AC06-76RLO 1830, the U.S. Environmental Protection Agency under Cooperative Agreement CR822062-01-0 to Battelle Memorial Institute, and internal research and development funds of the Battelle Memorial Institute. Pacific Northwest National Laboratory is operated for the U.S. Department of Energy by Battelle.

\section{References}

Ljungkvist, G.M. and Nordlinder, R.G. (1995). A field method for sampling benzene in end-exhaled air. Am. Ind. Hyg. Assoc. J. 56, 693-697. 
Kenny, D.V., and Kelly, T.J. (1991). Apparatus and method for analysis of expired breath. U.S. Patent 5,042,501. August, 1991.

Thrall, K.D., and Kenny, D.V. (1998). Technologies for measuring recent exposures: Volatile chemicals and the E2R monitor. IN: Biomarkers: Medical and Workplace Applications (Mendelsohn, M.L., Mohr, L.C. and Peeters, J.P., Eds.), Joseph Henry Press, National Academy of Sciences, Washington, D.C., Pp. 87-97.

Wallace, L.A. (1989). Major sources of benzene exposure. Environ. Health Perspect. 82, 165-169.

Thrall, K.D., Callahan, P.J., Weitz, K.K., Edwards, J.A., Brinkman, M.C., and Kenny, D.V. (2001). Design and evaluation of a breath-analysis system for biological monitoring of volatile compounds. Am. Ind. Hyg. Assoc. J. VV, pppppp. 


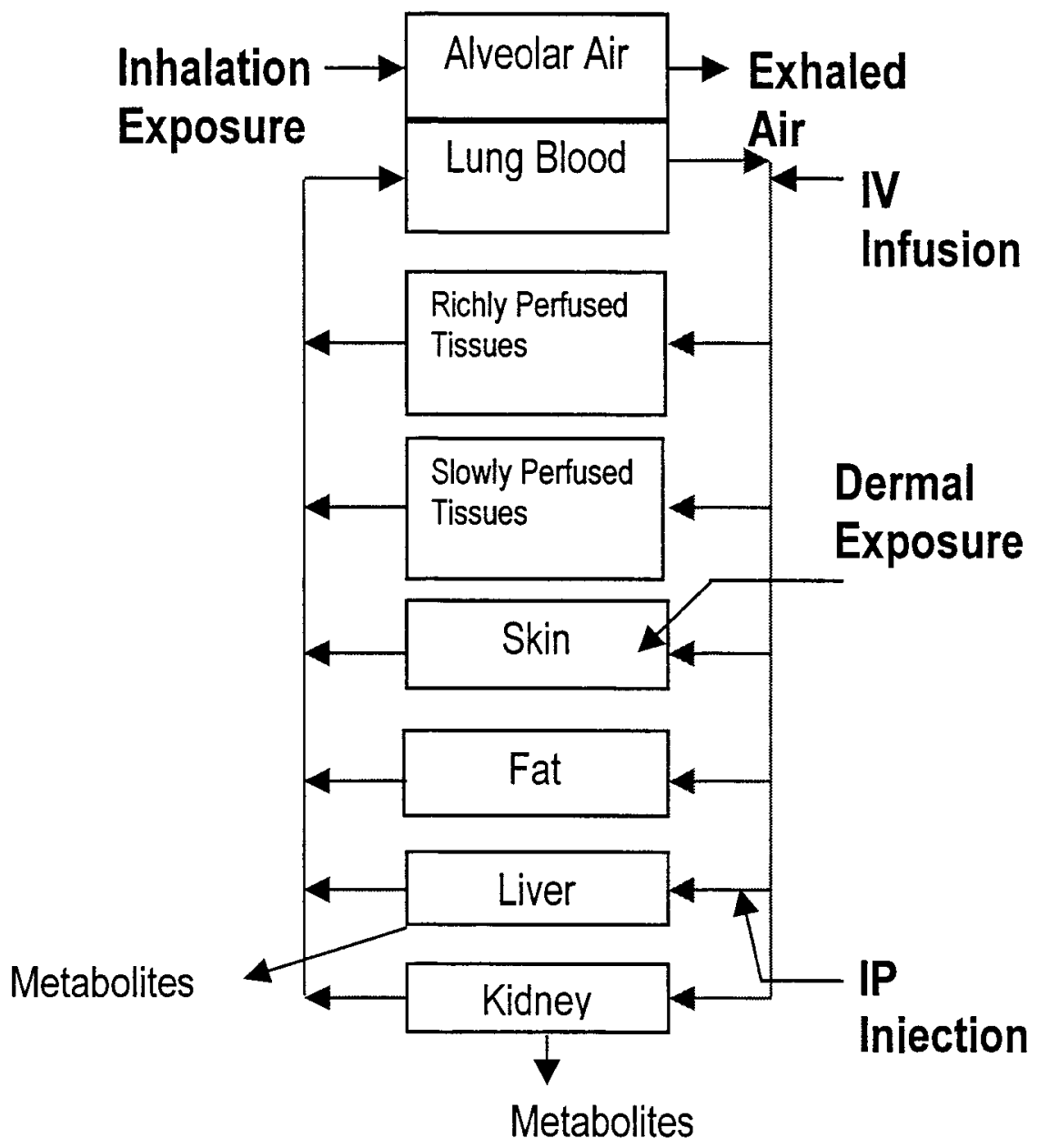

Figure 1: General structure of a PBPK model used to describe pharmacokinetics, tracking uptake from an oral, intravenous injection, inhalation, or dermal exposure, distribution into tissues of concern, metabolism, and elimination of the parent compound and/or metabolites in exhaled breath, blood, urine, and feces. 


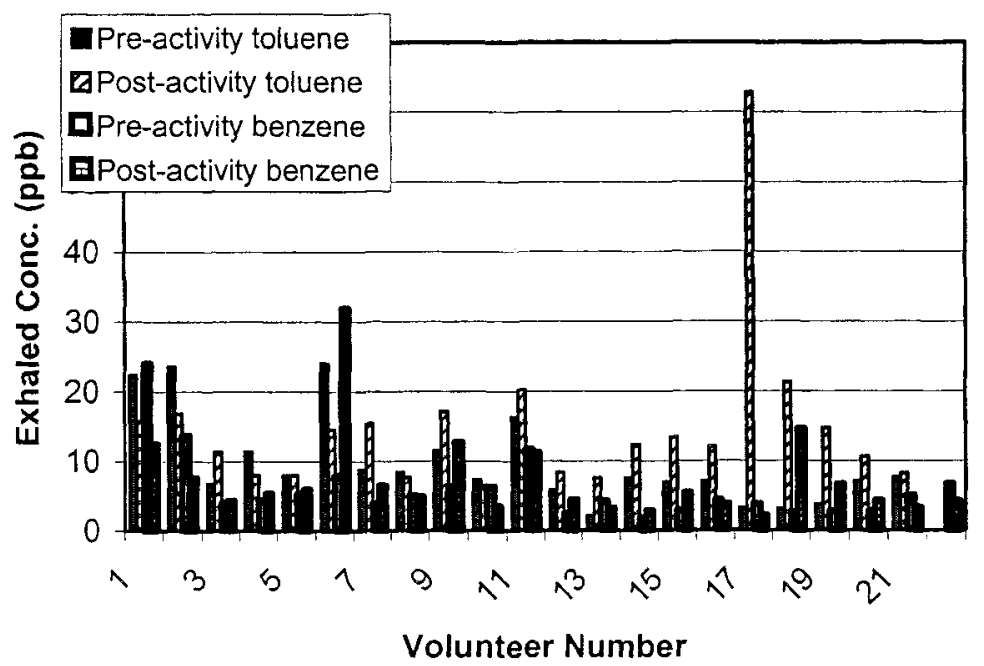

Figure 2: Comparison of pre-and post-activity measurements of toluene and benzene in the exhaled breath (in ppb) of volunteers participating in the field study at a waste incinerator.

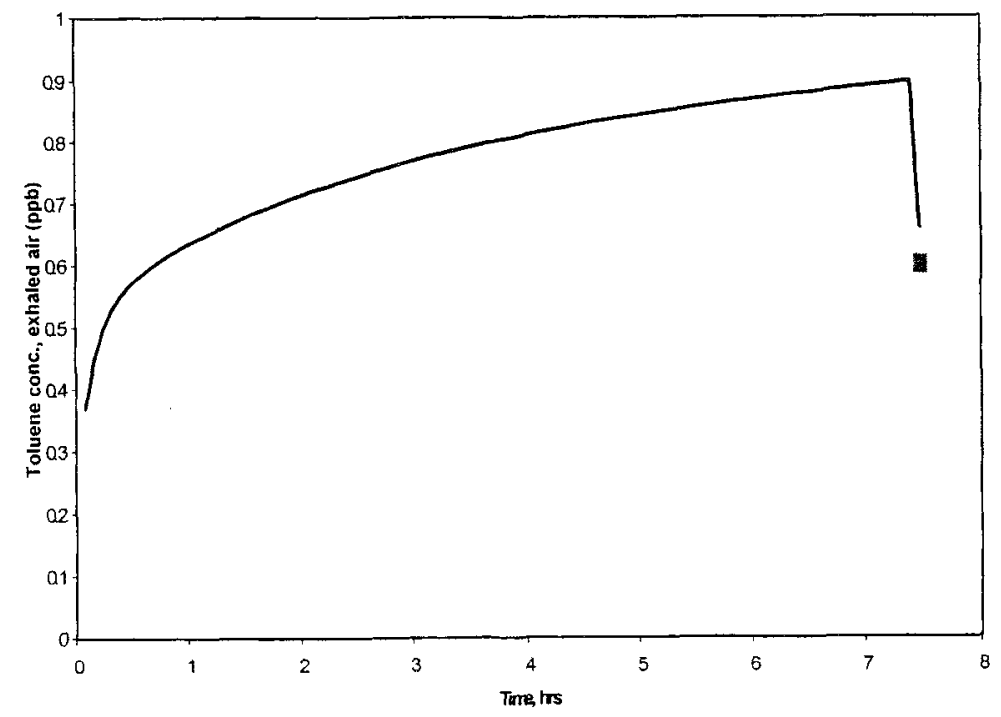

Figure 3. Comparison of the toluene PBPK model simulation of the concentration of toluene exhaled (in ppb) from a volunteer (2-3c) exposed at the time interval and level indicated by analysis of a Tenax sample cartridge (line) and the actual exhaled breath measurement (point). 